\title{
Gut Microbiome in Psoriasis: An Updated Review
}

\author{
Mariusz Sikora ${ }^{1, *} \mathbb{0}$, Albert Stec ${ }^{2}$, Magdalena Chrabaszcz ${ }^{1}$, Aleksandra Knot ${ }^{2}$, \\ Anna Waskiel-Burnat ${ }^{1}$, Adriana Rakowska ${ }^{1}$, Malgorzata Olszewska ${ }^{1}$ and Lidia Rudnicka ${ }^{1}$ \\ 1 Department of Dermatology, Medical University of Warsaw, 02-091 Warsaw, Poland; \\ chrabaszcz.magda@gmail.com (M.C.); waskiel.a@gmail.com (A.W.-B.); adriana.rakowska@gmail.com (A.R.); \\ malgorzataolszewska@yahoo.com (M.O.); lidiarudnicka@gmail.com (L.R.) \\ 2 Student Research Committee, Department of Dermatology, Medical University of Warsaw, \\ 02-091 Warsaw, Poland; albertmstec@gmail.com (A.S.); ola.knot@gmail.com (A.K.) \\ * Correspondence: msikora@wum.edu.pl
}

Received: 8 May 2020; Accepted: 8 June 2020; Published: 12 June 2020

\begin{abstract}
Background: A growing body of evidence highlights that intestinal dysbiosis is associated with the development of psoriasis. The gut-skin axis is the novel concept of the interaction between skin diseases and microbiome through inflammatory mediators, metabolites and the intestinal barrier. The objective of this study was to synthesize current data on the gut microbial composition in psoriasis. (2) Methods: We conducted a systematic review of studies investigating intestinal microbiome in psoriasis, using the PRISMA checklist. We searched MEDLINE, EMBASE, and Web of Science databases for relevant published articles (2000-2020). (3) Results: All of the 10 retrieved studies reported alterations in the gut microbiome in patients with psoriasis. Eight studies assessed alphaand beta-diversity. Four of them reported a lack of change in alpha-diversity, but all confirmed significant changes in beta-diversity. At the phylum-level, at least two or more studies reported a lower relative abundance of Bacteroidetes, and higher Firmicutes in psoriasis patients versus healthy controls. (4) Conclusions: There is a significant association between alterations in gut microbial composition and psoriasis; however, there is high heterogeneity between studies. More unified methodological standards in large-scale studies are needed to understand microbiota's contribution to psoriasis pathogenesis and its modulation as a potential therapeutic strategy.
\end{abstract}

Keywords: microbiome; psoriasis; gut-skin axis; gut barrier; systematic review

\section{Introduction}

Psoriasis is a common and chronic dermatological disease considered as a systemic inflammatory disorder [1]. Subclinical gut inflammation [2] and intestinal barrier dysfunction [3] reported in patients with psoriasis gave rise to the concept of the gut-skin axis. A growing body of evidence indicates that gut microbiota have a critical role in the regulation of metabolism [4], the immune system [5] and intestinal permeability [6]. The distortion in the biodiversity and composition of the gut microbiota, known as gut dysbiosis, has been linked to metabolic syndrome [7], inflammatory arthritis [8], depression [9], cardiovascular disease [10], inflammatory bowel diseases [11], which all are psoriasis comorbidities. Preclinical investigations provide evidence for the role of the gut microbiome in psoriasis pathogenesis. In mice with an experimental model of psoriasis induced by imiquimod, oral treatment with broad-spectrum antibiotic reduces the severity of skin inflammation through downregulation of Th17 immune response $[12,13]$. These results are supported by clinical observations based on a case series showing improvement in psoriatic skin lesions after antibiotic treatment [14], modulation of gut microbiota by probiotics [15] or fecal microbial transplantation [16].

In recent years, advancements in next-generation sequencing technologies have led to a thorough exploration of the intestinal microbiota composition [17]. However, the association of psoriasis with gut 
dysbiosis is mainly based on limited studies with small number of patients involved. Thus, the study aims to conduct an up-to-date systematic review of available gut microbiome composition studies in psoriasis, identify current methodological inconsistencies and outline directions for future research.

\section{Materials and Methods}

The systematic review was performed according the Preferred Reporting Items for Systematic Reviews and Meta-Analyses (PRISMA) guidelines [18]. The primary outcome measure of our study was a comparison of relative abundances of bacterial phyla, families, and genera in the gut microbiome of patients with psoriasis. Discrepancies between investigators at every stage of review were thoroughly discussed by all authors until a consensus was reached.

\subsection{Search Strategy}

Studies were identified by searching electronic databases and scanning reference lists of articles. We searched four electronic databases from their inception to March 2020: MEDLINE (via PubMed), Embase (via OvidSP), Web of Science Core Collection, and Scopus. To identify studies comparing gut microbiome composition in patients with psoriasis and normal healthy controls the combination of the following keywords was included: "psoriasis", "psoriatic arthritis", "microbiome", "microbiota", "bacteria", "dysbiosis", "gut", "gastrointestinal", "intestine", "stool", "fecal". Reference lists of the retrieved articles and relevant reviews were manually searched to identify further manuscripts not captured by the electronic searches.

\subsection{Inclusion/Exclusion Criteria}

The eligible inclusion criteria were presented as follows:

1. Subjects aged 18 years or older;

2. Human case-control studies investigating the association between gut microbiota and psoriasis

3. usage of culture-independent, high-throughput sequencing methods for gut microbiota quantification;

4. Articles published in English.

We excluded from the analysis: review papers, conference abstracts, case reports, expert opinions, editorials, and studies using animal models.

\subsection{Study Selection}

Four authors (M.S., A.S., M.C., A.K.) independently reviewed the titles and abstracts of all papers retrieved by the search strategy. Relevant full-text articles were evaluated for fulfilling the inclusion and exclusion criteria.

\subsection{Quality Assessment}

The quality of included studies was rated according to the Newcastle-Ottawa Scale (NOS), which is a valid tool for quality assessment of case-control studies. The scale evaluates three categories:

1. Selection (adequate case definition, representativeness of the cases, selection of controls, definition of controls);

2. Comparability (factors that the study controlled for by design or analysis to improve the comparability of baseline characteristics of cases and controls);

3. Exposure (ascertainment of exposure, same method of ascertainment for cases and controls, nonresponse rate).

\subsection{Data Extraction}

Six authors (M.S., A.S., M.C., A.K., A.W.-B., A.R.) in a pair-wise manner extracted the following data from the eligible studies: first author, publication year, country where the study was conducted, 
participant characteristics (sample size, age, sex, current treatment), sample materials, DNA extraction method, 16S rRNA variable region, sequencing platform, data analysis platform, reference sequences database employed in the studies, microbial diversity, alterations in gut microbiota in patients with psoriasis.

\section{Results}

\subsection{Search Results and Study Characteristics}

Ten records were included for the final analysis. The details of the article selection process are shown in Figure 1.
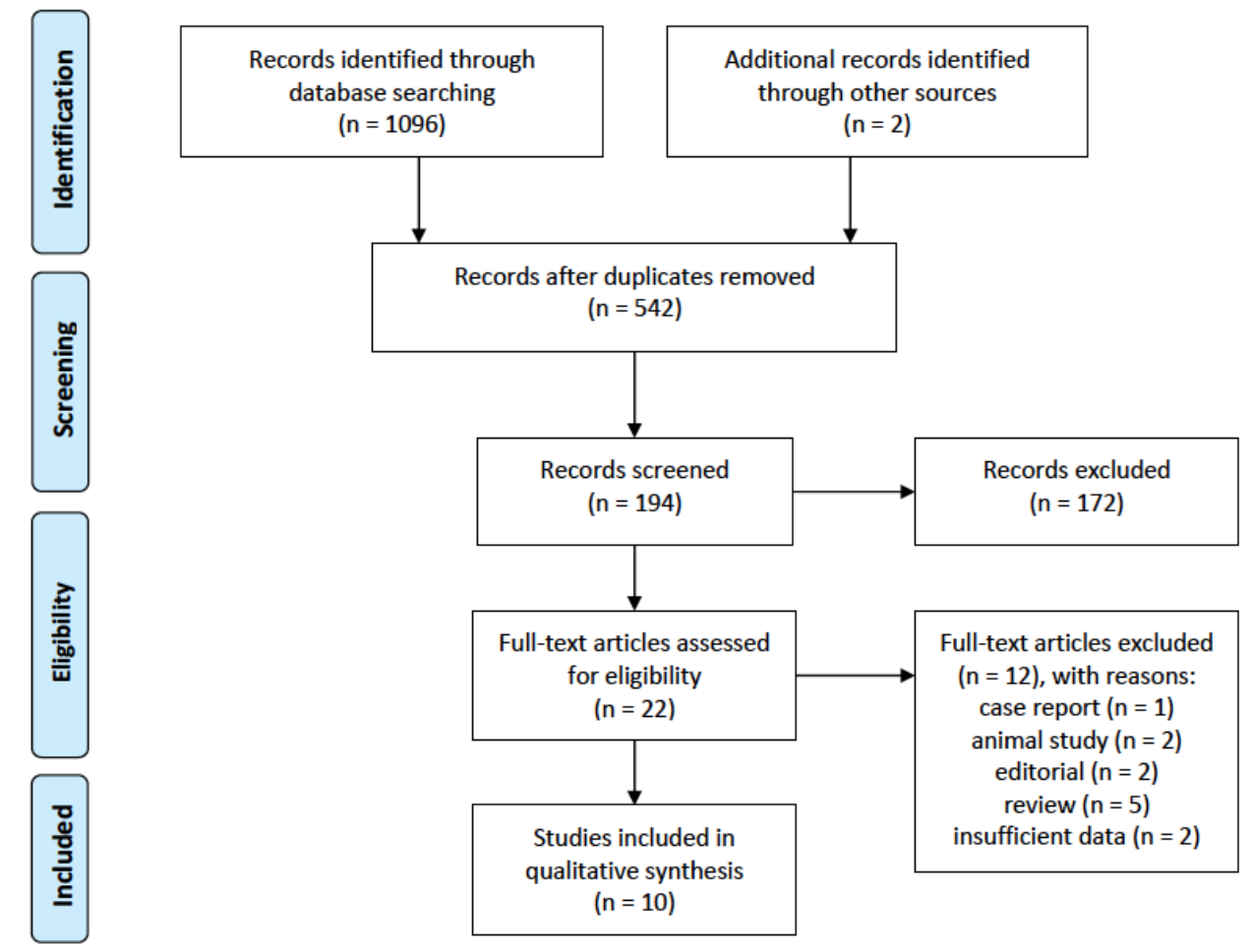

Figure 1. PRISMA flow diagram of study selection for inclusion in the systematic review; PRISMA-Preferred Reporting Items for Systematic Reviews and Meta-Analyses.

All eligible studies were published between 2015 and 2019 [19-28]. The general characteristics of the included studies are provided in Table 1. The studies were conducted in seven countries: China (two studies), Spain (two studies), Taiwan (two studies), Israel (one study), Netherland (one study), Egypt (one study), and the US (one study). The total sample size of included studies was 431 (299 patients with psoriasis and 254 healthy controls). Codoner et al. [24] compared psoriatic patients with a cohort of over 300 healthy individuals extracted from the Human Microbiome Project. This study was excluded from the age and sex analysis because the age range as well as gender distribution data for control group were not reported. The remaining studies were sex- and age-matched. The mean age was similar between compared groups and varied from 39.4 to 52.7 in patients with psoriasis and 40.4 to 52.9 in controls. Male patients constituted $56.6 \%$ of the studied population ( $57.5 \%$ in psoriasis group and $55.5 \%$ in control group). 
Table 1. General characteristic of the included studies.

\begin{tabular}{|c|c|c|c|c|c|c|c|c|c|}
\hline Study & Country & $\begin{array}{l}\text { Case } \\
\text { Number }\end{array}$ & $\begin{array}{c}\text { Case Age } \\
(\text { Mean } \pm \text { SD) }\end{array}$ & $\begin{array}{c}\text { Case } \\
\text { Gender } \\
\text { Men, }(\%)\end{array}$ & $\begin{array}{l}\text { Antipsoriatic } \\
\text { Therapy }\end{array}$ & $\begin{array}{l}\text { Control } \\
\text { Number }\end{array}$ & $\begin{array}{l}\text { Control Age } \\
(\text { Mean } \pm \text { SD) }\end{array}$ & $\begin{array}{c}\text { Control } \\
\text { Gender } \\
\text { Men, }(\%)\end{array}$ & NOS \\
\hline $\begin{array}{c}\text { Yeh et al., } 2019 \\
\text { [19] }\end{array}$ & Taiwan & 34 & $\begin{array}{c}\text { SEC: } 51 \pm 12 \\
\text { UST: } \\
48.4 \pm 12.7\end{array}$ & $25(73.5 \%)$ & $\begin{array}{l}\text { No treatment } \\
\text { except for } \\
\text { topical agents * }\end{array}$ & 12 & $48.4 \pm 13.3$ & $10(83.3 \%)$ & 8 \\
\hline $\begin{array}{c}\text { Shapiro et al., } \\
2019 \text { [20] }\end{array}$ & Israel & 24 & $52.7 \pm 11.6$ & $16(66.7 \%)$ & $\begin{array}{c}\text { Topical } \\
\text { treatment (22) } \\
\text { Biologics (2) }\end{array}$ & 22 & $43.9 \pm 12.7$ & $16(72.7 \%)$ & 8 \\
\hline $\begin{array}{l}\text { Huang et al., } \\
2019 \text { [21] }\end{array}$ & China & 35 & $52.1 \pm 3.0$ & $22(62.9 \%)$ & NR & 27 & $52.9 \pm 1.5$ & $16(59.3 \%)$ & 6 \\
\hline $\begin{array}{l}\text { Hidalgo- } \\
\text { Cantabrana et al., } \\
2019 \text { [22] }\end{array}$ & Spain & 19 & $49 \pm 11$ & $12(63.2 \%)$ & $\begin{array}{l}\text { No treatment } \\
\text { except for } \\
\text { topical } \\
\text { corticosteroids }\end{array}$ & 20 & $43 \pm 11$ & $5(25 \%)$ & 6 \\
\hline $\begin{array}{l}\text { Chen et al., } \\
2018 \text { [23] }\end{array}$ & Taiwan & 32 & $42.8 \pm 12.6$ & $25(78.1 \%)$ & $\begin{array}{c}\text { Phototherapy } \\
\text { (8) } \\
\text { DMARDs/ } \\
\text { Biologics (20) }\end{array}$ & 64 & $44.2 \pm 10.8$ & $\begin{array}{c}50 \\
(78.1 \%)\end{array}$ & 7 \\
\hline $\begin{array}{c}\text { Codoner et al., } \\
2018 \text { [24] }\end{array}$ & Spain & 52 & $41.2 \pm 14.4$ & $25(48.1 \%)$ & NR & NR & NR & NR & 4 \\
\hline $\begin{array}{c}\text { Tan et al., } 2018 \\
{[25]}\end{array}$ & China & 14 & $47.5 \pm 4.7$ & $10(71.4 \%)$ & NR & 14 & $40.4 \pm 2.5$ & $8(57.1 \%)$ & 7 \\
\hline $\begin{array}{l}\text { Eppinga et al., } \\
2016 \text { [26] }\end{array}$ & Netherlands & s 29 & $46 \pm 14.0$ & $12(41.4 \%)$ & $\begin{array}{c}\text { No treatment } \\
(27) \\
\text { DMARDs (2) }\end{array}$ & 33 & $41 \pm 14.9$ & $10(30.3 \%)$ & 6 \\
\hline $\begin{array}{l}\text { Massallat et al., } \\
2016 \text { [27] }\end{array}$ & Egypt & 45 & $42.3 \pm 10$ & $18(40 \%)$ & NR & 45 & $44.2 \pm 7.1$ & $20(44.4 \%)$ & 8 \\
\hline $\begin{array}{l}\text { Scher et al., } \\
2015 \text { [28] }\end{array}$ & US & 15 & 39.4 & $7(46.7 \%)$ & $\begin{array}{l}\text { No systemic } \\
\text { treatment }\end{array}$ & 17 & 42.2 & $6(35.3 \%)$ & 6 \\
\hline
\end{tabular}

All of the included studies used stool samples for gut microbiota profiling (sample and methodology characteristics of the included studies are summarized in Table 2). In five studies, the samples were frozen immediately after collection and remained frozen until DNA extraction. Other studies shipped the samples on ice $(n=3)$, and used either a nucleotide stabilizer before freezing $(n=1)$ or shipped the samples at ambient temperature within $48 \mathrm{~h}(\mathrm{n}=1)$. The majority of studies used different DNA extraction kits with QIAamp DNA Stool Mini Kit (Qiagen, Hilden, Germany) and PowerSoil DNA Extraction Kits (MoBio, Carlsbad, CA) used in several studies: five and two, respectively.

Eight studies have employed 16S rRNA gene sequencing, although with different variable regions for DNA amplification: region V1-V2 [28], region V2-V3 [22], region V3-V4 [19,23,24], region V4 [20,25], region V4-V5 [21]. Eppinga et al. [26] compared the abundance of Faecalibacterium prausnitzii and Escherichia coli in the fecal samples using quantitative PCR, while Masallat et al. [27] analyzed abundance of Firmicutes, Bacteroidetes and Actinobacteria. The most commonly used sequencing platform was the Illumina MiSeq platform. Data analysis pipelines were QIIME, UPARSE and USEARCH. Reference sequence databases used in the studies included the Silva database, Greengenes database, and Ribosomal Database Project. 
Table 2. Sample and methodology characteristics of the included studies.

\begin{tabular}{|c|c|c|c|c|c|c|c|c|}
\hline Study & Sample & $\begin{array}{c}\text { Sample } \\
\text { Transport }\end{array}$ & $\begin{array}{c}\text { DNA } \\
\text { Extraction }\end{array}$ & $\begin{array}{l}\text { Microbiota } \\
\text { Analysis } \\
\text { Technique }\end{array}$ & $\begin{array}{l}\text { Sequencing } \\
\text { Target }\end{array}$ & $\begin{array}{l}\text { Sequencing } \\
\text { Platform }\end{array}$ & $\begin{array}{c}\text { Data } \\
\text { Analysis } \\
\text { Platform }\end{array}$ & $\begin{array}{l}\text { Reference } \\
\text { Sequences } \\
\text { Database }\end{array}$ \\
\hline $\begin{array}{l}\text { Yeh et al., } \\
2019 \text { [19] }\end{array}$ & stool & $\begin{array}{l}\text { DNA stabilizer, } \\
\text { Immediate } \\
\text { freezing, } \\
\text { Transport on ice }\end{array}$ & $\begin{array}{l}\text { QIAamp } \\
\text { DNA } \\
\text { Stool } \\
\text { Mini Kit }\end{array}$ & $\begin{array}{l}\text { 16S rRNA } \\
\text { gene } \\
\text { sequencing }\end{array}$ & V3-V4 & $\begin{array}{l}\text { Ilumnia } \\
\text { MiSeq } \\
\text { platform }\end{array}$ & QIIME & $\begin{array}{c}\text { Kyoto } \\
\text { Encyclopedia } \\
\text { of Genes and } \\
\text { Genomies } \\
\text { database }\end{array}$ \\
\hline $\begin{array}{l}\text { Shapiro et al., } \\
2019 \text { [20] }\end{array}$ & stool & $\begin{array}{l}\text { Immediate } \\
\text { freezing }\end{array}$ & $\begin{array}{l}\text { PowerSoil } \\
\text { HTP } 96 \\
\text { Kit }\end{array}$ & $\begin{array}{l}\text { 16S rRNA } \\
\text { gene } \\
\text { sequencing }\end{array}$ & $\mathrm{V} 4$ & $\begin{array}{l}\text { Ilumnia } \\
\text { MiSeq } \\
\text { platform }\end{array}$ & QIIME & $\begin{array}{c}\text { Greengenes } \\
\text { database }\end{array}$ \\
\hline $\begin{array}{l}\text { Huang et al., } \\
2019 \text { [21] }\end{array}$ & stool & Transport on ice & $\begin{array}{l}\text { PowerSoil } \\
\text { HTP } 96 \\
\text { Kit }\end{array}$ & $\begin{array}{l}\text { 16S rRNA } \\
\text { gene } \\
\text { sequencing }\end{array}$ & V4-V5 & $\begin{array}{l}\text { Ilumnia } \\
\text { MiSeq } \\
\text { platform }\end{array}$ & UPARSE & $\begin{array}{l}\text { National } \\
\text { Center for } \\
\text { Biotechnology } \\
\text { Information } \\
\text { Sequence } \\
\text { Read Archive } \\
\text { database }\end{array}$ \\
\hline $\begin{array}{l}\text { Hidalgo- } \\
\text { Cantabrana et al. } \\
2019 \text { [22] }\end{array}$ & l., stool & $\begin{array}{l}\text { Immediate } \\
\text { freezing }\end{array}$ & $\begin{array}{l}\text { QIAamp } \\
\text { DNA } \\
\text { Stool } \\
\text { Mini Kit }\end{array}$ & $\begin{array}{l}\text { 16S rRNA } \\
\text { gene } \\
\text { sequencing }\end{array}$ & V2-V3 & $\begin{array}{l}\text { Ion Gene } \\
\text { Studio S5 } \\
\text { sequencer }\end{array}$ & QIIME & $\begin{array}{c}\text { SILVA } \\
\text { database }\end{array}$ \\
\hline $\begin{array}{l}\text { Chen et al., } \\
2018 \text { [23] }\end{array}$ & stool & Transport on ice & $\begin{array}{l}\text { QIAamp } \\
\text { DNA } \\
\text { Stool } \\
\text { Mini Kit }\end{array}$ & $\begin{array}{l}\text { 16S rRNA } \\
\text { gene } \\
\text { sequencing }\end{array}$ & V3-V4 & $\begin{array}{l}\text { Ilumnia } \\
\text { MiSeq } \\
\text { platform }\end{array}$ & UPARSE & $\begin{array}{c}\text { Greengenes } \\
\text { database }\end{array}$ \\
\hline $\begin{array}{l}\text { Codoner et al., } \\
2018 \text { [24] }\end{array}$ & stool & $\begin{array}{l}\text { Immediate } \\
\text { freezing }\end{array}$ & $\begin{array}{l}\text { QIAamp } \\
\text { DNA } \\
\text { Stool } \\
\text { Mini Kit }\end{array}$ & $\begin{array}{l}\text { 16S rRNA } \\
\text { gene } \\
\text { sequencing }\end{array}$ & V3-V4 & $\begin{array}{l}\text { Ilumnia } \\
\text { MiSeq } \\
\text { platform }\end{array}$ & QIIME & $\begin{array}{l}\text { National } \\
\text { Center for } \\
\text { Biotechnology } \\
\text { Information }\end{array}$ \\
\hline $\begin{array}{l}\text { Tan et al., } \\
2018 \text { [25] }\end{array}$ & stool & $\begin{array}{l}\text { Immediate } \\
\text { freezing }\end{array}$ & $\begin{array}{l}\text { E.Z.N.A. } \\
\text { stool } \\
\text { DNA Kit }\end{array}$ & $\begin{array}{l}\text { 16S rRNA } \\
\text { gene } \\
\text { sequencing }\end{array}$ & V4 & $\begin{array}{l}\text { Ilumnia } \\
\text { MiSeq } \\
\text { platform }\end{array}$ & USEARCH & $\begin{array}{c}\text { Ribosomal } \\
\text { Database } \\
\text { Project (RDP) }\end{array}$ \\
\hline $\begin{array}{c}\text { Eppinga et al., } \\
2016 \text { [26] }\end{array}$ & stool & $\begin{array}{c}\text { Ambient } \\
\text { temperature }\end{array}$ & NR & $\begin{array}{l}\text { real-time } \\
\text { quantitative } \\
\text { PCR }\end{array}$ & - & $\begin{array}{c}\text { quantitative } \\
\text { PCR }\end{array}$ & - & - \\
\hline $\begin{array}{l}\text { Massallat et al., } \\
2016 \text { [27] }\end{array}$ & stool & NR & $\begin{array}{l}\text { QIAamp } \\
\text { DNA } \\
\text { Stool } \\
\text { Mini Kit }\end{array}$ & $\begin{array}{c}\text { real-time } \\
\text { quantitative } \\
\text { PCR }\end{array}$ & - & $\begin{array}{c}\text { quantitative } \\
\text { PCR }\end{array}$ & - & - \\
\hline $\begin{array}{l}\text { Scher et al., } \\
2015 \text { [28] }\end{array}$ & stool & NR & NR & $\begin{array}{l}\text { 16S rRNA } \\
\text { gene } \\
\text { pyro-sequen }\end{array}$ & $\mathrm{g}^{\mathrm{V} 1-\mathrm{V} 2}$ & $\begin{array}{c}454 \text { GS FLX } \\
\text { Titanium } \\
\text { platform }\end{array}$ & Mothur & $\begin{array}{c}\text { SILVA } \\
\text { database }\end{array}$ \\
\hline
\end{tabular}

NR-not reported.

\subsubsection{Gut Microbiome Diversity in Psoriasis}

Eight of ten studies assessed alpha-diversity. The majority of them fail to show significant changes $[19,20,23,25]$, two reported lower alpha-diversity $[22,28]$ and one greater diversity in psoriasis [24]. Huang et al. demonstrated lower community richness in psoriasis with similar level of diversity [21]. All studies used Shannon's Diversity Index to assess alpha-diversity. Four studies additionally assess alpha-diversity with Simpson or Chao1; two studies used the abundance-based Coverage Estimator (ACE) and Faith phylogenetic diversity index. Observed species richness and rarefaction curve were also mentioned.

Beta-diversity was assessed in all studies that performed 16S rRNA gene sequencing, all of which reported a significant difference between psoriasis and healthy controls. However, a study by Chen et al. [23] reported that differences reached statistical significance only for psoriatic patients with a BMI lower than 25. Employed estimates of beta-diversity included Bray-Curtis dissimilarity distance, principal coordinate analysis, and weighted and unweighted UniFrac distances. An overview of alphaand beta-diversity in psoriasis is provided in Table 3. 
Table 3. Alpha- and beta-diversity of gut microbiome in psoriasis compared with healthy controls.

\begin{tabular}{|c|c|c|c|c|}
\hline Study & $\begin{array}{l}\text { Indices of } \\
\alpha \text {-Diversity }\end{array}$ & $\begin{array}{l}\text { Gut Microbiota } \alpha \text {-Diversity in } \\
\text { Psoriasis }\end{array}$ & $\begin{array}{l}\text { Indices of } \\
\beta \text {-Diversity }\end{array}$ & $\begin{array}{c}\text { Gut Microbiota } \beta \text {-Diversity in } \\
\text { Psoriasis }\end{array}$ \\
\hline Yeh et al. [19] & Shannon index, Simpson index & No differences were observed & $\begin{array}{l}\text { UniFrac analysis (weighted } \\
\text { and unweighted analyses), } \\
\text { Bray Curtis index }\end{array}$ & Significant difference \\
\hline $\begin{array}{l}\text { Shapiro et al. } \\
\text { [20] }\end{array}$ & Shannon index, rarefaction curves & No differences were observed & $\begin{array}{l}\text { UniFrac analysis (weighted } \\
\text { and unweighted analyses) }\end{array}$ & Significant difference \\
\hline $\begin{array}{l}\text { Huang et al. } \\
\text { [21] }\end{array}$ & $\begin{array}{l}\text { Shannon index, Simpson index, } \\
\text { ACE index, } \\
\text { Chao1 index }\end{array}$ & $\begin{array}{c}\text { Shannon and Simpson } \\
\text { indexes-no differences } \\
\text { ACE and Chao } \\
\text { indexes-decreased in psoriasis }\end{array}$ & $\begin{array}{l}\text { PCA based on the Bray-Curtis } \\
\text { dissimilarity distance }\end{array}$ & Significant difference \\
\hline $\begin{array}{l}\text { Hidalgo- } \\
\text { Cantabrana et } \\
\text { al. [22] }\end{array}$ & $\begin{array}{c}\text { Shannon index, } \\
\text { Chao1 index, } \\
\text { Faith's phylogenetic diversity index }\end{array}$ & Lower diversity in psoriasis & unweighted Unifrac analysis & Significant difference \\
\hline $\begin{array}{l}\text { Chen et al. } \\
\text { [23] }\end{array}$ & $\begin{array}{l}\text { Shannon index, Simpson index, } \\
\text { Chao1 index, } \\
\text { number of observed OTUs }\end{array}$ & No differences were observed & $\begin{array}{l}\text { UniFrac analysis (weighted } \\
\text { and unweighted analyses), } \\
\text { Bray Curtis index }\end{array}$ & $\begin{array}{l}\text { Significant difference (psoriasis } \\
\text { patients with BMI }<25 \text { ) }\end{array}$ \\
\hline $\begin{array}{l}\text { Scher et al. } \\
\text { [28] }\end{array}$ & $\begin{array}{c}\text { Shannon index, Faith's phylogenetic } \\
\text { diversity index }\end{array}$ & Lower diversity in psoriasis & unweighted Unifrac analysis & Significant difference \\
\hline
\end{tabular}

\subsubsection{Gut Microbiome Alterations in Psoriasis}

All studies confirmed the association of psoriasis and gut microbiota dysbiosis. At the phylum level, Bacteroidetes had a lower relative abundance and Firmicutes a higher relative abundance in patients with psoriasis than in healthy controls $[20,22,23]$. However, in a study by Huang et al., Bacteroidetes was reported to be increased and Firmicutes decreased in psoriasis [21]. Additionally, two studies showed decreased amounts of Proteobacteria in psoriatic cohort [20,22]. Studies for Actinobacteria provide conflicting results—an increase in two studies $[20,22]$ and decrease in the other two studies $[27,28]$.

At the family level, the relative abundance of Ruminococcaceae, Lachnospiraceae, Clostridiales Family XIII, Peptostreptococcaceae, Enterococcaceae, Coriobacteriaceae, and Eggerthellaceae was increased in psoriasis, whereas Prevotellaceae, Barnesiellaceae, Tannerellaceae, Rikenellaceae, Porphyromonadaceae, Marinifilaceae, S24-7, Lactobacillaceae, Streptococcaceae, Pasteurellaceae, Burkholderiaceae, Desulfovibrionaceae, Victivallaceae, and Verrucomicrobiaceae decreased. There were conflicting results for Bacteroidaceae, Erysipelotrichaceae, Veillonellaceae and Bifidobacteriaceae. Some studies reported these families to be decreased in psoriasis $[22,23,28]$ while others showed their increase [22,25].

At the genus level, Paraprevotella, Barnesiella, Alistipes, Allobaculum, Coprobacillus, Carnobacterium, Granulicatella, Rothia, Gordonibacter, Thermus were found to be decreased. Following genera were relatively increased in psoriasis: Ruminococcus, Subdoligranulum, Blautia, Coprococcus, Dorea, Christensenella, Streptococcus, Lactococcus, Enterococcus, Bacillus, Collinsella, Slackia. Divergent findings were reported for Bacteroides, Parabacteroides, Faecalibacterium, Lachnospira, Akkermansia, Sutterella, and Bifidobacterium.

At the species level, Prevotella copri, Faecalibacterium prausnitzii and Akkermiansia muciniphila were found to be significantly decreased, while Ruminococcus gnavus, Dorea formicigenerans, Clostridium citroniae, Escherichia coli, and Collinsella aerofaciens were increased in patients with psoriasis compared to control group. However, these alterations have not been confirmed in more than one study. Details indicating the altered bacteria are shown in Table 4. 
Table 4. Gut microbiota alterations in psoriasis.

\begin{tabular}{|c|c|c|c|c|c|}
\hline Phylum & Class & Order & Family & Genus & Species \\
\hline \multirow[t]{10}{*}{$\begin{array}{c}\text { Bacteroidetes } \\
\uparrow[21] \\
\downarrow[20,22,23]\end{array}$} & Bacteroidia & Bacteroidales & $\begin{array}{c}\text { Bacteroidaceae } \\
\uparrow[25] \\
\downarrow[22,23]\end{array}$ & $\begin{array}{c}\text { Bacteroides } \\
\uparrow[25] \\
\downarrow[22,24]\end{array}$ & \\
\hline & & & $\begin{array}{c}\text { Prevotellaceae } \\
\downarrow[22,23]\end{array}$ & Prevotella & $\begin{array}{c}\text { Prevotella copri } \\
\downarrow[20]\end{array}$ \\
\hline & & & & $\begin{array}{c}\text { Paraprevotella } \\
\downarrow[20,22]\end{array}$ & \\
\hline & & & $\begin{array}{l}\text { Barnesiellaceae } \\
\downarrow[22]\end{array}$ & $\begin{array}{l}\text { Barnesiella } \\
\quad \downarrow[22]\end{array}$ & \\
\hline & & & $\begin{array}{c}\text { Tannerellaceae } \\
\downarrow[22]\end{array}$ & & \\
\hline & & & $\begin{array}{l}\text { Rikenellaceae } \\
\quad \downarrow[22]\end{array}$ & $\begin{array}{l}\text { Alistipes } \\
\downarrow[22]\end{array}$ & \\
\hline & & & Tannerellaceae & $\begin{array}{c}\text { Parabacteroides } \\
\uparrow[21] \\
\downarrow[22,28]\end{array}$ & \\
\hline & & & $\begin{array}{c}\text { Porphyromonadaceae } \\
\downarrow \downarrow 28]\end{array}$ & & \\
\hline & & & $\begin{array}{l}S 24-7 \\
\downarrow[25]\end{array}$ & & \\
\hline & & Marinilabiliales & $\begin{array}{c}\text { Marinifilaceae } \\
\downarrow[22]\end{array}$ & & \\
\hline \multirow[t]{12}{*}{$\begin{array}{c}\text { Firmicutes } \\
\uparrow[20,22,23] \\
\downarrow[21]\end{array}$} & Clostridia & Clostridiales & $\begin{array}{c}\text { Ruminococcaceae } \\
\uparrow[22,23]\end{array}$ & $\begin{array}{c}\text { Faecalibacterium } \\
\uparrow[20,24] \\
\downarrow[22]\end{array}$ & $\begin{array}{c}\text { Faecalibacterium } \\
\text { prausnitzii } \\
\downarrow[26]\end{array}$ \\
\hline & & & & $\begin{array}{c}\text { Ruminococcus } \\
\uparrow[20,22]\end{array}$ & $\begin{array}{c}\text { Ruminococcus } \\
\text { gnavus } \\
\uparrow[20]\end{array}$ \\
\hline & & & & $\begin{array}{c}\text { Subdoligranulum } \\
\uparrow[22]\end{array}$ & \\
\hline & & & $\begin{array}{c}\text { Lachnospiraceae } \\
\quad \uparrow[22,23]\end{array}$ & $\begin{array}{l}\text { Lachnospira } \\
\quad \uparrow[21] \\
\downarrow[20]\end{array}$ & \\
\hline & & & & $\begin{array}{c}\text { Blautia } \\
\uparrow[20,22]\end{array}$ & \\
\hline & & & & $\begin{array}{c}\text { Coprococcus } \\
\uparrow[20]\end{array}$ & \\
\hline & & & & $\begin{array}{l}\text { Dorea } \\
\uparrow[20]\end{array}$ & $\begin{array}{c}\text { Dorea } \\
\text { formicigenerans } \\
\uparrow[20]\end{array}$ \\
\hline & & & $\begin{array}{c}\text { Clostridiales } \\
\text { Family XIII } \\
\quad \uparrow[22]\end{array}$ & & \\
\hline & & & $\begin{array}{c}\text { Peptostreptococcaceae } \\
\uparrow[22]\end{array}$ & & \\
\hline & & & Christensenellaceae & $\begin{array}{c}\text { Christensenella } \\
\uparrow[20]\end{array}$ & \\
\hline & & & Clostridiaceae & Clostridium & $\begin{array}{c}\text { Clostridium } \\
\text { citroniae } \\
\uparrow[25]\end{array}$ \\
\hline & Erysipelotrichia & $\begin{array}{c}\text { Erysipelotrichales } \\
\downarrow \downarrow[28]\end{array}$ & $\begin{array}{c}\text { Erysipelotrichaceae } \\
\uparrow[22] \\
\downarrow[28]\end{array}$ & $\begin{array}{l}\text { Allobaculum } \\
\quad \downarrow[22]\end{array}$ & \\
\hline
\end{tabular}


Table 4. Cont.

\begin{tabular}{|c|c|c|c|c|c|}
\hline Phylum & Class & Order & Family & Genus & Species \\
\hline & & & & $\begin{array}{c}\text { Coprobacillus } \\
\downarrow[28]\end{array}$ & \\
\hline & Bacilli & Lactobacillales & $\begin{array}{c}\text { Lactobacillaceae } \\
\downarrow[22]\end{array}$ & & \\
\hline & & & $\begin{array}{c}\text { Streptococcaceae } \\
\downarrow[22]\end{array}$ & $\begin{array}{c}\text { Streptococcus } \\
\uparrow[21]\end{array}$ & \\
\hline & & & & $\begin{array}{l}\text { Lactococcus } \\
\quad \uparrow[21]\end{array}$ & \\
\hline & & & Carnobacteriaceae & $\begin{array}{c}\text { Carnobacterium } \\
\downarrow[21]\end{array}$ & \\
\hline & & & & $\begin{array}{c}\text { Granulicatella } \\
\downarrow[21]\end{array}$ & \\
\hline & & & $\begin{array}{c}\text { Enterococcaceae } \\
\uparrow[25]\end{array}$ & $\begin{array}{l}\text { Enterococcus } \\
\quad \uparrow[25]\end{array}$ & \\
\hline & & Bacillales & Bacillaceae & $\begin{array}{c}\text { Bacillus } \\
\uparrow[21]\end{array}$ & \\
\hline & Negativicutes & Veillonellales & $\begin{array}{c}\text { Veillonellaceae } \\
\quad \uparrow[25] \\
\downarrow[22]\end{array}$ & & \\
\hline \multirow[t]{2}{*}{$\begin{array}{l}\text { Verrucomicrobia } \\
\quad \downarrow[25]\end{array}$} & $\begin{array}{c}\text { Verrucomicrobiae } \\
\downarrow[25]\end{array}$ & $\begin{array}{l}\text { Verrucomicrobiales } \\
\qquad[25]\end{array}$ & Akkermansiaceae & $\begin{array}{l}\text { Akkermansia } \\
\uparrow[24] \\
\downarrow[25]\end{array}$ & $\begin{array}{c}\text { Akkermiansia } \\
\text { muciniphila } \\
\downarrow[25]\end{array}$ \\
\hline & & & $\begin{array}{l}\text { Verrucomicrobiaceae } \downarrow \\
{[25]}\end{array}$ & & \\
\hline \multirow[t]{5}{*}{$\begin{array}{c}\text { Proteobacteria } \\
\quad \downarrow[20,22]\end{array}$} & Gammaproteobacteria & Enterobacterales & Enterobacteriaceae & Escherichia & $\begin{array}{c}\text { Escherichia coli } \\
\uparrow[26]\end{array}$ \\
\hline & & Pasteurellales & $\begin{array}{c}\text { Pasteurellaceae } \\
\downarrow[22]\end{array}$ & & \\
\hline & Betaproteobacteria & Burkholderiales & $\begin{array}{c}\text { Burkholderiaceae } \\
\downarrow[22]\end{array}$ & & \\
\hline & & & Sutterellaceae & $\begin{array}{c}\text { Sutterella } \\
\uparrow[21] \\
\downarrow[20]\end{array}$ & \\
\hline & Deltaproteobacteria & Desulfovibrionales & $\begin{array}{c}\text { Desulfovibrionaceae } \\
\downarrow[22]\end{array}$ & & \\
\hline \multirow[t]{5}{*}{$\begin{array}{c}\text { Actinobacteria } \\
\quad \uparrow[20,22] \\
\downarrow[27,28]\end{array}$} & $\begin{array}{c}\text { Actinobacteria } \\
\downarrow[28]\end{array}$ & $\begin{array}{c}\text { Bifidobacteriales } \\
\downarrow[28]\end{array}$ & $\begin{array}{c}\text { Bifidobacteriaceae } \\
\uparrow[22] \\
\downarrow[28]\end{array}$ & $\begin{array}{c}\text { Bifidobacterium } \\
\uparrow[20,22] \\
\downarrow[28]\end{array}$ & \\
\hline & & Micrococcales & Micrococcaceae & $\begin{array}{l}\text { Rothia } \\
\downarrow[21]\end{array}$ & \\
\hline & Coriobacteriia & Coriobacteriales & $\begin{array}{c}\text { Coriobacteriaceae } \\
\uparrow[22]\end{array}$ & $\begin{array}{c}\text { Collinsella } \\
\uparrow[20,22]\end{array}$ & $\begin{array}{c}\text { Collinsella } \\
\text { aerofaciens } \\
\uparrow[20]\end{array}$ \\
\hline & & Eggerthellales & $\begin{array}{c}\text { Eggerthellaceae } \\
\uparrow[22]\end{array}$ & $\begin{array}{l}\text { Slackia } \\
\uparrow[22]\end{array}$ & \\
\hline & & & & $\begin{array}{c}\text { Gordonibacter } \\
\downarrow[21]\end{array}$ & \\
\hline Lentisphaerae & Lentisphaeria & Victivallales & $\begin{array}{l}\text { Victivallaceae } \\
\quad \downarrow[22]\end{array}$ & & \\
\hline $\begin{array}{l}\text { Deinococcus- } \\
\text { Thermus }\end{array}$ & Deinococci & Thermales & Thermaceae & $\begin{array}{c}\text { Thermus } \\
\downarrow[21]\end{array}$ & \\
\hline $\begin{array}{l}\text { Tenericutes } \\
\quad \downarrow[25]\end{array}$ & $\begin{array}{l}\text { Mollicutes } \\
\quad \downarrow[25]\end{array}$ & & & & \\
\hline
\end{tabular}




\subsubsection{Changes in Gut Microbiota after Antipsoriatic Treatment}

To date, only one study compared changes in the composition of the pre-treatment and post-treatment intestinal microbiome. Yeh et al. [19] analyzed stool samples from 12 healthy individuals and 34 psoriatic patients at baseline, 3 and 6 months after biological treatment with secukinumab (24 patients) or ustekinumab (10 patients). Secukinumab treatment caused more profound alterations in gut microbiome, such as an increase in the relative abundance of phylum Proteobacteria and decrease in Bacteroidetes and Firmicutes. At other levels of taxonomic classification, treatment with secukinumab led to an increase in Citrobacter and a decrease in Aeromonas, Bacteroides, Ruminococcus torques at the genera level. At the family level, Enterobacteriaceae and Pseudomonadaceae were significantly increased, while Aeromonadaceae decreased. In contrast, there was no significant change in gut microbiome after ustekinumab treatment, where only genus Coprococcus significantly increased after 6 months.

\subsubsection{Quality of the Evidence}

All included studies were assessed for quality according to NOS and scored between 4 to 8 points (Table 1).

\section{Discussion}

Recent advances in genome sequencing and bioinformatic analysis have enabled a greater understanding of the complex host-microbiome associations. However, since the first publication on gut microbiome differences in psoriasis by Scher et al. [28], there have been more opinion papers and editorials on the significance of the gut-skin axis than laboratory investigations. This paper provides a detailed and comprehensive systematic review regarding gut microbiome in patients with psoriasis. To cover a wider range of microbiome alterations we extracted the data of every available bacterial group using the lowest taxonomic level of each included study.

In this review, we found that most of the studies reported no significant differences in alpha-diversity between patients with psoriasis and healthy subjects based on similar indices. Comparable findings have also been shown in other chronic inflammatory diseases, namely multiple sclerosis [29], ankylosing spondylitis [30] and ulcerative colitis [31]. Although alpha-diversity remained unchanged in psoriasis, all studies revealed significant beta-diversity differences. Diversity indices are one of the tools enable to characterized microbiome [32]. Alpha-diversity describes the variety of the microbial community in a single sample, taking into account the number of different taxa and their relative abundances. Beta-diversity measures diversity of microbial communities' composition between different samples. Microbial community may experience a complete shift in composition, where no taxa are shared, but can still have similar alpha-diversity index [33]. Given all the above findings, it can be speculated that gut dysbiosis in psoriasis may be a result of differential abundance of bacteria instead of the quantity of bacterial species.

We presented several taxa that differed in their relative abundance in psoriasis. For instance, a reduction in Bacteroides and Proteobacteria with increased proportions of Firmicutes and Actinobacteria, at phylum level was reported in more than one study. These four phyla constitute $>98 \%$ of the gut microbiota. Therefore, the Firmicutes/Bacteroidetes (F/B) ratio is considered as an important marker of gut microbiota state. Several studies have shown that an altered $\mathrm{F} / \mathrm{B}$ ratio in gut microbiome is associated with psoriasis comorbidities, such as cardiovascular diseases [34], obesity [35], insulin resistance [36] and nonalcoholic fatty liver disease [37]. Only one study by Huang et al. reported opposite changes in Bacteroides and Firmicutes [21]. This dissimilarity partially may be related to a small number of participants and very diverse group, which consisted of people with plaque psoriasis, pustular psoriasis, erythrodermic psoriasis and psoriatic arthritis.

The tendencies for the change of Firmicutes and Bacteroidetes in patients with psoriasis are also present at the lower taxonomic levels. Families Bacteroidaceae and Prevotellaceae are two important subgroups in phylum Bacteroides. While alterations in Bacteroidaceae and Bacteroides have been confirmed 
in psoriasis, there is no agreement about the direction of these changes. Similarly, the role of these bacteria is also controversial. Bacteroides fragilis, can produce enterotoxins responsible for inflammation and impairment of the intestinal barrier [38]. Conversely, non-toxigenic Bacteroides fragilis exerts beneficial effects by production of short-chain fatty acids and polysaccharide A with anti-inflammatory properties [38]. Prevotella copri, another species belonging to the same phylum, was found to be decreased in psoriasis. This finding is in opposite to other inflammatory diseases-ankylosing spondylitis [39] or rheumatoid arthritis [40]-where there is an increased abundance of P. copri. This shift in microbial composition may be modulated by the duration of the disease as well as by using conventional or biological therapy $[39,40]$. A problem that remains to be resolved is whether $P$. copri is beneficial or detrimental, as studies report conflicting results on metabolic and inflammatory actions [41]. The pro- or anti-inflammatory effect may be dependent on the diet and the fact that P. copri is not a monotypic species but composed of four distinct clades [42].

Regarding the second important phylum, Firmicutes, at least two studies have found increased abundance of families Ruminococcaceae and Lachnospiraceae, with depletion of Faecalibacterium prausnitzii and augmentation of Ruminococcus gnavus at the species level. F. prausnitzii metabolites exert a protective effect on the gut barrier and inhibit the activation of the NF- $\kappa \mathrm{B}$, altering the pro-inflammatory response [43]. The depletion of F. prausnitzii has been associated with inflammatory disorders, such as inflammatory bowel disease or ankylosing spondylitis [26,39]. On the other hand, R. gnavus produces an inflammatory polysaccharide and contributes to gut barrier dysfunction. Its increased abundance was observed in inflammatory bowel disease, spondyloarthritis, eczema and coronary artery disease [44].

It is still not clear whether psoriasis is an effect or a cause of the observed disbalance between beneficial and pathogenic microbes. These complex gut microbiome-host interactions include destruction of tight junction proteins and intestinal barrier integrity, initiation and maintenance of inflammation, and changes in metabolites production $[3,45]$. Another proposed mechanism underlying the link between gut dysbiosis and skin changes implies the modulation of $\mathrm{T}$ cells differentiation and function with an imbalance between Th17 and T regulatory (Treg) cells. In experimental model of psoriasis, it has been shown that alterations in the intestinal microbiota may promote Th17-mediated skin inflammation [12,13]. In this context, the study by Yeh et al. [19] provides very interesting results, showing significantly greater changes in the gut microbiome of patients with psoriasis treated with secukinumab (interleukin-17 inhibitor) compared to ustekinumab (inhibitor of interleukin-12 and -23). On the other hand, in the same study, the baseline composition of the microbiome differed significantly between responders and non-responders to secukinumab treatment [19]. In complex interactions in the gut-skin axis, psoriasis severity should also be considered in addition to the microbiome and intestinal barrier. It has been shown that Psoriasis Activity and Severity Index (PASI) positively correlates with the blood concentration of intestinal barrier damage biomarkers [46,47]. Much less is known about the potential relationship between the composition of gut microbiome and the severity of psoriasis. Among the analyzed studies, only two assessed such a potential relationship. Masallat et al. [27] found positive correlation between PASI and Firmicutes/Bacteroidetes ratio as well as a negative correlation between PASI and Actinobacteria phylum. On the contrary, according to a study by Chen et al. [23], disease activity assessed by PASI score did not significantly affect the abundance profile of gut microbiome among patients with psoriasis.

This review clearly demonstrated that despite significant differences in taxa between psoriasis and control groups, there is a lack of consistency in the results concerning microbial diversity, relative abundance, or directionality of differences. The results' heterogeneity may be explained, to a certain degree, by differences in study population, design and methodology. Psoriasis is a heterogeneous disorder with a broad spectrum of clinical presentations. Similar to other chronic inflammatory diseases, disease activity, disease duration, comorbidities and treatment, may be also responsible for microbiota changes [30,31,39]. The low sample size, ranging from 14 to 52 patients with psoriasis, makes it impossible to assess the above factors for their confounding influence on microbiome composition. Unlike the fixed and unchangeable human genome, the gut microbiome 
is highly dynamic and it can be modulated by age, sex, geographical background and diet [32,48]. None of the analyzed studies were multicenter, which may account for inter-study differences as seven countries were represented. Another mentioned variable is diet, but most studies did not examine dietary habits. For future studies, it is highly recommended to provide a complete assessment of nutrient intake by food diaries or food frequency questionnaires.

We found different methodologies in all phases, starting from sample collection transportation and storage, through DNA extraction and sequencing to computational analysis. Microbiota profiling requires particular care in terms of methodology choices, while each step can be a potential source of bias [49]. All the included studies investigated the gut microbiome using fecal samples. This approach carries a serious limitation; it does not allow to determine changes in the microbiome across the intestine. There is also difference in composition of luminal- and mucosal-associated microbiota [50]. Additionally, it may not be clear whether an identified taxon maintains pathogenic role or is just nonviable and transient. To reduce this bias, there is a need for the simultaneous application of several sampling techniques, such as intestine bioptates, mucosal-luminal interface aspirates or colonic lavage. 16S rRNA gene profiling is the most common approach to the study of a microbiome. Only two articles limited their search of the gut microbiome a priori to specific taxa: F. prausnitzii and E. coli [26] or Firmicutes, Bacteroidetes and Actinobacteria [27]. Even among the eight studies that employed 16S rRNA gene sequencing, different selection of targeted variable region will bias identification and quantification [51].

\section{Conclusions}

Several studies demonstrate differences in the microbiome composition of patients with psoriasis. However, no specific changes in results, methodological inconsistencies and unaddressed confounders make the translation into clinical practice difficult. Useful information may be obtained from a meta-analysis, but, currently, due to the studies' high heterogeneity and limited number of publications, this approach seems to be impractical. Instead, we highlighted commonalities and identify existing problems. An in-depth understanding of the complex gut-skin axis requires the standardization of study protocols and a unified methodological approach. Finally, large-scale, longitudinal studies with a multiomics assessment would give detailed insight into the microbiome-host interactions and allow to identify targeted therapies.

Author Contributions: Conceptualization, M.S., A.S., M.O. and L.R.; methodology, M.S., A.S., M.C., A.K., A.W.-B., A.R., M.O. and L.R.; writing-original draft preparation, M.S.; writing-review and editing, A.S., M.C., A.K., A.W.-B., A.R., M.O. and L.R.; funding acquisition, A.S., M.C., A.K. All authors have read and agreed to the published version of the manuscript.

Funding: This research was funded by the Polish Ministry of Science and Higher Education, grant number MNiSW/2020/220/DIR/NN4.

Conflicts of Interest: The authors declare no conflict of interest.

\section{References}

1. Korman, N.J. Management of psoriasis as a systemic disease: What is the evidence? Br. J. Dermatol. 2019. [CrossRef] [PubMed]

2. Adarsh, M.B.; Dogra, S.; Vaiphei, K.; Vaishnavi, C.; Sinha, S.K.; Sharma, A. Evaluation of subclinical gut inflammation using faecal calprotectin levels and colonic mucosal biopsy in patients with psoriasis and psoriatic arthritis. Br. J. Dermatol. 2019, 181, 401-402. [CrossRef] [PubMed]

3. Sikora, M.; Chrabaszcz, M.; Maciejewski, C.; Zaremba, M.; Waskiel, A.; Olszewska, M.; Rudnicka, L. Intestinal barrier integrity in patients with plaque psoriasis. J. Dermatol. 2018, 45, 1468-1470. [CrossRef]

4. Lin, S.; Wang, Z.; Lam, K.L.; Zeng, S.; Tan, B.K.; Hu, J. Role of intestinal microecology in the regulation of energy metabolism by dietary polyphenols and their metabolites. Food Nutr. Res. 2019, 63. [CrossRef] [PubMed] 
5. Spencer, S.P.; Fragiadakis, G.K.; Sonnenburg, J.L. Pursuing Human-Relevant Gut Microbiota-Immune Interactions. Immunity 2019, 51, 225-239. [CrossRef] [PubMed]

6. Chelakkot, C.; Ghim, J.; Ryu, S.H. Mechanisms regulating intestinal barrier integrity and its pathological implications. Exp. Mol. Med. 2018, 50, 103. [CrossRef] [PubMed]

7. Vallianou, N.; Stratigou, T.; Christodoulatos, G.S.; Dalamaga, M. Understanding the Role of the Gut Microbiome and Microbial Metabolites in Obesity and Obesity-Associated Metabolic Disorders: Current Evidence and Perspectives. Curr. Obes. Rep. 2019, 8, 317-332. [CrossRef] [PubMed]

8. Scher, J.U.; Littman, D.R.; Abramson, S.B. Microbiome in Inflammatory Arthritis and Human Rheumatic Diseases. Arthritis Rheumatol. 2016, 68, 35-45. [CrossRef]

9. Stevens, B.R.; Roesch, L.; Thiago, P.; Russell, J.T.; Pepine, C.J.; Holbert, R.C.; Raizada, M.K.; Triplett, E.W. Depression phenotype identified by using single nucleotide exact amplicon sequence variants of the human gut microbiome. Mol. Psychiatry 2020. [CrossRef]

10. Kitai, T.; Tang, W.H.W. Gut microbiota in cardiovascular disease and heart failure. Clin. Sci. 2018, $132,85-91$. [CrossRef]

11. Nishida, A.; Inoue, R.; Inatomi, O.; Bamba, S.; Naito, Y.; Andoh, A. Gut microbiota in the pathogenesis of inflammatory bowel disease. Clin. J. Gastroenterol. 2018, 11, 1-10. [CrossRef]

12. Zakostelska, Z.; Malkova, J.; Klimesova, K.; Rossmann, P.; Hornova, M.; Novosadova, I.; Stehlikova, Z.; Kostovcik, M.; Hudcovic, T.; Stepankova, R.; et al. Intestinal Microbiota Promotes Psoriasis-Like Skin Inflammation by Enhancing Th17 Response. PLoS ONE 2016, 11, e0159539. [CrossRef]

13. Stehlikova, Z.; Kostovcikova, K.; Kverka, M.; Rossmann, P.; Dvorak, J.; Novosadova, I.; Kostovcik, M.; Coufal, S.; Srutkova, D.; Prochazkova, P.; et al. Crucial Role of Microbiota in Experimental Psoriasis Revealed by a Gnotobiotic Mouse Model. Front. Microbiol. 2019, 10, 236. [CrossRef]

14. Walecka, I.; Olszewska, M.; Rakowska, A.; Slowinska, M.; Sicinska, J.; Piekarczyk, E.; Kowalska-Oledzka, E.; Goralska, B.; Rudnicka, L. Improvement of psoriasis after antibiotic therapy with cefuroxime axetil. J. Eur. Acad. Dermatol. Venereol. 2009, 23, 957-958. [CrossRef] [PubMed]

15. Szanto, M.; Dozsa, A.; Antal, D.; Szabo, K.; Kemeny, L.; Bai, P. Targeting the gut-skin axis-Probiotics as new tools for skin disorder management? Exp. Dermatol. 2019, 28, 1210-1218. [CrossRef]

16. Yin, G.; Li, J.F.; Sun, Y.F.; Ding, X.; Zeng, J.Q.; Zhang, T.; Peng, L.H.; Yang, Y.S.; Zhao, H. Fecal microbiota transplantation as a novel therapy for severe psoriasis. Zhonghua Nei Ke Za Zhi 2019, 58, 782-785. [CrossRef]

17. Sacchetti, L.; Nardelli, C. Gut microbiome investigation in celiac disease: From methods to its pathogenetic role. Clin. Chem. Lab. Med. 2020, 58, 340-349. [CrossRef]

18. Moher, D.; Liberati, A.; Tetzlaff, J.; Altman, D.G.; Group, P. Preferred reporting items for systematic reviews and meta-analyses: The PRISMA statement. BMJ 2009, 339, b2535. [CrossRef]

19. Yeh, N.L.; Hsu, C.Y.; Tsai, T.F.; Chiu, H.Y. Gut Microbiome in Psoriasis is Perturbed Differently During Secukinumab and Ustekinumab Therapy and Associated with Response to Treatment. Clin. Drug Investig. 2019, 39, 1195-1203. [CrossRef]

20. Shapiro, J.; Cohen, N.A.; Shalev, V.; Uzan, A.; Koren, O.; Maharshak, N. Psoriatic patients have a distinct structural and functional fecal microbiota compared with controls. J. Dermatol. 2019, 46, 595-603. [CrossRef]

21. Huang, L.; Gao, R.; Yu, N.; Zhu, Y.; Ding, Y.; Qin, H. Dysbiosis of gut microbiota was closely associated with psoriasis. Sci. China Life Sci. 2019, 62, 807-815. [CrossRef] [PubMed]

22. Hidalgo-Cantabrana, C.; Gomez, J.; Delgado, S.; Requena-Lopez, S.; Queiro-Silva, R.; Margolles, A.; Coto, E.; Sanchez, B.; Coto-Segura, P. Gut microbiota dysbiosis in a cohort of patients with psoriasis. Br. J. Dermatol. 2019, 181, 1287-1295. [CrossRef] [PubMed]

23. Chen, Y.J.; Ho, H.J.; Tseng, C.H.; Lai, Z.L.; Shieh, J.J.; Wu, C.Y. Intestinal microbiota profiling and predicted metabolic dysregulation in psoriasis patients. Exp. Dermatol. 2018, 27, 1336-1343. [CrossRef] [PubMed]

24. Codoner, F.M.; Ramirez-Bosca, A.; Climent, E.; Carrion-Gutierrez, M.; Guerrero, M.; Perez-Orquin, J.M.; Horga de la Parte, J.; Genoves, S.; Ramon, D.; Navarro-Lopez, V.; et al. Gut microbial composition in patients with psoriasis. Sci. Rep. 2018, 8, 3812. [CrossRef]

25. Tan, L.; Zhao, S.; Zhu, W.; Wu, L.; Li, J.; Shen, M.; Lei, L.; Chen, X.; Peng, C. The Akkermansia muciniphila is a gut microbiota signature in psoriasis. Exp. Dermatol. 2018, 27, 144-149. [CrossRef]

26. Eppinga, H.; Sperna Weiland, C.J.; Thio, H.B.; van der Woude, C.J.; Nijsten, T.E.; Peppelenbosch, M.P.; Konstantinov, S.R. Similar Depletion of Protective Faecalibacterium prausnitzii in Psoriasis and Inflammatory Bowel Disease, but not in Hidradenitis Suppurativa. J. Crohns Colitis 2016, 10, 1067-1075. [CrossRef] 
27. Masallat, D.; Moemen, D.; State, A.F. Gut bacterial microbiota in psoriasis: A case control study. Afr. J. Microbiol. Res. 2016, 10,1337-1343.

28. Scher, J.U.; Ubeda, C.; Artacho, A.; Attur, M.; Isaac, S.; Reddy, S.M.; Marmon, S.; Neimann, A.; Brusca, S.; Patel, T.; et al. Decreased bacterial diversity characterizes the altered gut microbiota in patients with psoriatic arthritis, resembling dysbiosis in inflammatory bowel disease. Arthritis Rheumatol. 2015, 67, 128-139. [CrossRef]

29. Mirza, A.; Forbes, J.D.; Zhu, F.; Bernstein, C.N.; Van Domselaar, G.; Graham, M.; Waubant, E.; Tremlett, H. The multiple sclerosis gut microbiota: A systematic review. Mult. Scler. Relat. Disord. 2020, 37, 101427. [CrossRef]

30. Chen, Z.; Qi, J.; Wei, Q.; Zheng, X.; Wu, X.; Li, X.; Liao, Z.; Lin, Z.; Gu, J. Variations in gut microbial profiles in ankylosing spondylitis: Disease phenotype-related dysbiosis. Ann. Transl. Med. 2019, 7, 571. [CrossRef]

31. Pittayanon, R.; Lau, J.T.; Leontiadis, G.I.; Tse, F.; Yuan, Y.; Surette, M.; Moayyedi, P. Differences in Gut Microbiota in Patients With vs Without Inflammatory Bowel Diseases: A Systematic Review. Gastroenterology 2020, 158, 930-946.e931. [CrossRef]

32. Scepanovic, P.; Hodel, F.; Mondot, S.; Partula, V.; Byrd, A.; Hammer, C.; Alanio, C.; Bergstedt, J.; Patin, E.; Touvier, M.; et al. A comprehensive assessment of demographic, environmental, and host genetic associations with gut microbiome diversity in healthy individuals. Microbiome 2019, 7, 130. [CrossRef] [PubMed]

33. Wagner, B.D.; Grunwald, G.K.; Zerbe, G.O.; Mikulich-Gilbertson, S.K.; Robertson, C.E.; Zemanick, E.T.; Harris, J.K. On the Use of Diversity Measures in Longitudinal Sequencing Studies of Microbial Communities. Front. Microbiol. 2018, 9, 1037. [CrossRef] [PubMed]

34. Yoshida, N.; Yamashita, T.; Hirata, K.I. Gut Microbiome and Cardiovascular Diseases. Diseases 2018, 6, 56. [CrossRef] [PubMed]

35. Crovesy, L.; Masterson, D.; Rosado, E.L. Profile of the gut microbiota of adults with obesity: A systematic review. Eur. J. Clin. Nutr. 2020. [CrossRef] [PubMed]

36. Moreno-Indias, I.; Sanchez-Alcoholado, L.; Garcia-Fuentes, E.; Cardona, F.; Queipo-Ortuno, M.I.; Tinahones, F.J. Insulin resistance is associated with specific gut microbiota in appendix samples from morbidly obese patients. Am. J. Transl. Res. 2016, 8, 5672-5684. [PubMed]

37. Sobhonslidsuk, A.; Chanprasertyothin, S.; Pongrujikorn, T.; Kaewduang, P.; Promson, K.; Petraksa, S.; Ongphiphadhanakul, B. The Association of Gut Microbiota with Nonalcoholic Steatohepatitis in Thais. Biomed. Res. Int. 2018, 2018, 9340316. [CrossRef]

38. Valguarnera, E.; Wardenburg, J.B. Good Gone Bad: One Toxin Away From Disease for Bacteroides fragilis. J. Mol. Biol. 2020, 432, 765-785. [CrossRef]

39. Zhou, C.; Zhao, H.; Xiao, X.Y.; Chen, B.D.; Guo, R.J.; Wang, Q.; Chen, H.; Zhao, L.D.; Zhang, C.C.; Jiao, Y.H.; et al. Metagenomic profiling of the pro-inflammatory gut microbiota in ankylosing spondylitis. J. Autoimmun. 2019, 102360. [CrossRef]

40. Guerreiro, C.S.; Calado, A.; Sousa, J.; Fonseca, J.E. Diet, Microbiota, and Gut Permeability-The Unknown Triad in Rheumatoid Arthritis. Front. Med. 2018, 5, 349. [CrossRef]

41. De Filippis, F.; Pasolli, E.; Tett, A.; Tarallo, S.; Naccarati, A.; De Angelis, M.; Neviani, E.; Cocolin, L.; Gobbetti, M.; Segata, N.; et al. Distinct Genetic and Functional Traits of Human Intestinal Prevotella copri Strains Are Associated with Different Habitual Diets. Cell Host Microbe 2019, 25, 444-453.e443. [CrossRef] [PubMed]

42. Tett, A.; Huang, K.D.; Asnicar, F.; Fehlner-Peach, H.; Pasolli, E.; Karcher, N.; Armanini, F.; Manghi, P.; Bonham, K.; Zolfo, M.; et al. The Prevotella copri Complex Comprises Four Distinct Clades Underrepresented in Westernized Populations. Cell Host Microbe 2019, 26, 666-679.e667. [CrossRef] [PubMed]

43. Stefia, L.V.; Lee, J.; Patel, J.; de Sousa, S.R.; Legrand, J.; Rodero, M.; Burman, S.; Linedale, R.; Morrison, M.; Khosrotehrani, K. Secretome components from Faecalibacterium prausnitzii strains A2-165 and AHMP21 Modulate Cutaneous Wound Inflammation. J. Investig. Dermatol. 2020. [CrossRef]

44. Toya, T.; Corban, M.T.; Marrietta, E.; Horwath, I.E.; Lerman, L.O.; Murray, J.A.; Lerman, A. Coronary artery disease is associated with an altered gut microbiome composition. PLOS ONE 2020, 15, e227147. [CrossRef]

45. Ufnal, M.; Pham, K. The gut-blood barrier permeability - A new marker in cardiovascular and metabolic diseases? Med. Hypotheses 2017, 98, 35-37. [CrossRef] 
46. Sikora, M.; Chrabaszcz, M.; Waskiel-Burnat, A.; Rakowska, A.; Olszewska, M.; Rudnicka, L. Claudin-3-A new intestinal integrity marker in patients with psoriasis: Association with disease severity. J. Eur. Acad. Dermatol. Venereol. 2019, 33, 1907-1912. [CrossRef]

47. Sikora, M.; Stec, A.; Chrabaszcz, M.; Waskiel-Burnat, A.; Zaremba, M.; Olszewska, M.; Rudnicka, L. Intestinal Fatty Acid Binding Protein, a Biomarker of Intestinal Barrier, is Associated with Severity of Psoriasis. J. Clin. Med. 2019, 8, 1021. [CrossRef]

48. Knox, N.C.; Forbes, J.D.; Peterson, C.L.; Van Domselaar, G.; Bernstein, C.N. The Gut Microbiome in Inflammatory Bowel Disease: Lessons Learned From Other Immune-Mediated Inflammatory Diseases. Am. J. Gastroenterol. 2019, 114, 1051-1070. [CrossRef]

49. Panek, M.; Cipcic Paljetak, H.; Baresic, A.; Peric, M.; Matijasic, M.; Lojkic, I.; Vranesic Bender, D.; Krznaric, Z.; Verbanac, D. Methodology challenges in studying human gut microbiota-Effects of collection, storage, DNA extraction and next generation sequencing technologies. Sci. Rep. 2018, 8, 5143. [CrossRef]

50. Mottawea, W.; Butcher, J.; Li, J.; Abujamel, T.; Manoogian, J.; Mack, D.; Stintzi, A. The mucosal-luminal interface: An ideal sample to study the mucosa-associated microbiota and the intestinal microbial biogeography. Pediatr. Res. 2019, 85, 895-903. [CrossRef]

51. Castelino, M.; Eyre, S.; Moat, J.; Fox, G.; Martin, P.; Ho, P.; Upton, M.; Barton, A. Optimisation of methods for bacterial skin microbiome investigation: Primer selection and comparison of the 454 versus MiSeq platform. BMC Microbiol. 2017, 17, 23. [CrossRef]

(C) 2020 by the authors. Licensee MDPI, Basel, Switzerland. This article is an open access article distributed under the terms and conditions of the Creative Commons Attribution (CC BY) license (http://creativecommons.org/licenses/by/4.0/). 